\title{
Evolution of residual stress, free volume, and hardness in the laser shock peened Ti-based metallic glass
}

\author{
Liang Wang ${ }^{a}$, Lu Wanga, Zhihua Nie ${ }^{a}$, Yang Ren ${ }^{b}$, Yunfei Xue ${ }^{a^{*}}$, Ronghua Zhu ${ }^{c}$, \\ Haifeng Zhang ${ }^{d}$, and Huameng Fu ${ }^{d}$
}

${ }^{a}$ School of Materials Science and Engineering, Beijing Institute of Technology, Beijing 100081, China.

${ }^{\mathrm{b}}$ X-ray Science Division, Argonne National Laboratory, Argonne, IL 60439, USA

${ }^{c}$ AML, School of Aerospace, Tsinghua University, Beijing 100084, China

d Shenyang National Laboratory for Materials Science, Institute of Metal Research, Chinese Academy of Sciences, Shenyang 110016, China.

${ }^{*}$ Corresponding author at: School of Materials Science and Engineering, Beijing Institute of Technology, Beijing 100081, China. Tel.: +86 01068912709 ext. 107; Email address: xueyunfei@bit.edu.cn; (Yunfei Xue)

\begin{abstract}
Laser shock peening (LSP) with different cycles was performed on the Ti-based bulk metallic glasses (BMGs). The internal residual stress of the LSPed specimens was measured by high-energy X-ray diffraction (HEXRD) and the residual stress on the shocked surface was measured by scanning electron microscope/focused ion beam (SEM/FIB) instrument. The internal residual stress in the LSP impact direction (about $-170 \mathrm{MPa}$ ) is much lower than that perpendicular to the impact direction (about -350 $\mathrm{MPa}$ ), exhibiting anisotropy. The depth of the compressive stress zone increases from $400 \mu \mathrm{m}$ to $500 \mu \mathrm{m}$ with increasing LSP cycles. The highest residual stress on the shocked surface is about -750 MPa. LSP caused the free volume to increase and the maximum increase appeared after the first LSP process. Compared with the hardness $(567 \pm 7 \mathrm{HV})$ of the as-cast BMG, the hardness $(590 \pm 9 \mathrm{HV})$ on the shocked surface shows a hardening effect due to the hardening mechanism of compressive residual
\end{abstract}


stress; and the hardness ( $420 \pm 9 \mathrm{HV})$ on the longitudinal section shows a softening effect due to the softening mechanism of free volume.

\section{Keywords}

Bulk metallic glasses; laser shock peening; High energy X-ray diffraction; Residual stress; Free volume; Hardness.

\section{Introduction}

Bulk metallic glasses (BMGs) have received much attention in the past decades due to their unique mechanical properties. However, the application is limited in practice due to the low plasticity [1], which is commonly considered as a result of the formation of highly localized shear bands. It was proved that multiple shear bands and residual compressive stress $(\sigma)$ could be introduced and the plasticity was improved after the mechanical treatments, such as shot peening (SP) [2,3] and cold-rolling [4-6]. The multiple shear bands could effectively alleviate the shear band localization, and the residual compressive stress could hinder the propagation of the activated shear bands.

According to the models proposed by Spaepen and Argon [7,8], amounts of free volume were found to induced in shear bands during plastic deformation, and the free volume increase resulted in a decrease in hardness [9-12]. The residual compressive stress, however, could inhibit the propagation of shear bands and promote a hardening effect on BMGs $[13,14]$. Under the combined influence of free volume and residual stress, the structure evolution of BMGs is quite complicated. Stolpe et al. [6] found that although the free volume increased after cold-rolling, the hardness decreased only in the early stage of the deformation (true plastic strain $<0.1$ ), and then increased subsequently. However, Song et al. [5] found that the micro-hardness increased from $545 \mathrm{HV}$ to $600 \mathrm{HV}$ after cold-rolling the BMG (thickness reduction of $2.9 \%$ ). These inconsistent results show that accurate measurement of the free volume and residual stress are the prerequisites to understand the structure evolution of BMGs during deformation. The free volume could be calculated by measuring the structural relaxation enthalpy $(H)$ using a Differential scanning calorimetry (DSC) $[3,6,15]$, while 
the residual stress is difficult to measure due to the amorphous structure without any crystal lattice in BMGs.

There are two methods has been used to measure the residual stress in the BMGs: the scanning electron microscope/focused ion beam (SEM/FIB) instrument $[16,17]$ and the high energy X-ray diffraction (HEXRD) [18]. For the SEM/FIB instrument, a crack on the surface would be slit by FIB to release the stress, and the material around the crack would shift toward (for compressive stress) or away from (for tensile stress) the crack $[17,19]$. The displacement field is inferred by the digital image correlation (DIC) analysis and the stress on the surface before the FIB slitting could be calculated. For the HEXRD, it has been used to explore the atomic structure changes of metallic glasses after heating [20] and loading [18, 21-23]. The atomic elastic strain $(\varepsilon)$ at short and medium range scales in BMGs varies linearly with the loading stress in the elastic regime [21-23], since the $\varepsilon$ storing in the BMGs could shift the diffraction peak, when the X-ray passes through the BMGs, the shift could be measured and the internal stress within the BMGs could be obtained $[18,22]$. It could be found the SEM/FIB instrument is applicable for the measurement of residual stress on the surface, while the HEXRD could give the internal stress.

Recently, laser shock peening (LSP) was used as a new mechanical treatment technology to improve the plasticity of BMGs $[16,17,24-26]$ and the estimation of residual stress was attempted. Cao et al. successfully calculated the residual stress of the LSP treated (LSPed) BMG by SEM/FIB instrument, and the compression tests revealed that the plasticity was obviously increased [17]. In our previous work, it has been proved that the LSP could induce an effect region with thickness more than 500 $\mu \mathrm{m}[26]$, and the residual stress on the shocked surface could be more than $-700 \mathrm{MPa}$ (“-” means compressive stress) measured by SEM/FIB instrument [16].

The purpose of the present paper is to focus on the distribution of residual stress after different LSP cycles, and to investigate the effect of free volume and residual stress on the hardness of BMG. In the present paper, the internal residual stress of the LSPed 
BMG was measured by HEXRD, and the residual stress on the shocked surface was measured by SEM/FIB instrument.

\section{Experimental}

$\mathrm{Ti}_{32.8} \mathrm{Zr}_{30.2 \mathrm{Ni}}{ }_{3.3} \mathrm{Cu}_{9} \mathrm{Be}_{22.7}$ (at. \%) metallic glass plates were prepared by arc-melting the elemental metals (Ti-gettered argon atmosphere) followed by flip casting into a copper mold. The Young's modulus of the BMG was measured to be $90 \pm 3 \mathrm{MPa}$ using global ultra-phonic method. The plates were cut into specimens $\left(40 \times 7 \times 5 \mathrm{~mm}^{3}\right)$ using a water cooled low speed diamond wire saw. Prior to LSP, the as-cast specimens were polished to minimize the influence of surface roughness by SiC sandpapers (from \#400 to \#2000) and diamond polishing solution.

For the LSP process, the laser pulse with wavelength of $1064 \mathrm{~nm}$ was generated by Q-switched high power Nd-doped yttrium aluminum garnet (YAG) pulse laser. The power density of a single laser pulse is $25 \mathrm{GW} / \mathrm{cm}^{2}$ and the pulse duration is $30 \mathrm{~ns}$, the laser pulse energy was measured by an optical power meter (Coherent, type: Field Max II). The diameter of the laser beam is $3 \mathrm{~mm}$. To absorb laser energy and generate plasma, an aluminum foil with $0.12 \mathrm{~mm}$ thickness was covered on the specimen. A water layer with $2 \mathrm{~mm}$ thickness flowed over the surface of the aluminum foil to constrain the plasma. To investigate the LSP cycle effect on BMGs, 1 time treatment (LSP-1), 2 times treatment (LSP-2), and 3 times treatment (LSP-3) were processed on the specimens.

Differential scanning calorimetry (DSC) (NETZSCH STA 449F3) was employed to estimate the free volume change based on the measurement of enthalpy recovery at a heating rate of $20 \mathrm{~K} / \mathrm{min}$, the temperature range is $298 \mathrm{~K} \sim 873 \mathrm{~K}$. The size of the specimens for DSC is $3.2 \times 3.2 \times 0.3 \mathrm{~mm}^{3}$, each cycle number has 3 specimens for the DSC tests. Micro-hardness tests (SHY11-FM-700E) were performed on the shocked surface and longitudinal section (perpendicular to the shocked surface) of the LSPed specimens, with a loading force $(F)$ of $1.96 \mathrm{~N}$ and holding time of $10 \mathrm{~s}$. The Vickers hardness $\left(H_{v}\right)$ was determined by measuring the mean of the two diagonal lengths $(d)$, i.e. $H_{v}=0.1891 F / d^{2}$. However, due to the pileup generation on the surface during the hardness tests $[6,14]$, the real hardness $H_{r}$ was calculated by measuring the real area $A_{r}$ 
of the each hardness indentation, i.e. $H_{r}=0.1891 F / 2 A_{r}$. Each reported hardness corresponds to the average value of at least 5 indents.

The nanoindentation tests were performed on the shocked surface and the longitudinal section of the LSP-1 specimens by MTS Nano Indenter XP system with Berkovich triangular diamond tip, the loading rate is $0.5 \mathrm{mN} / \mathrm{s}$. The final load is 100 $\mathrm{mN}$. At least five indentations in each condition were performed. Atomic force microscopy (AFM) (MFP-3D-SA) was used to characterize the morphology of the indentations.

Structural studies were carried out using HEXRD at the 11-ID-C beam line (with a wavelength of $0.11165 \AA$ ) of the Advanced Photon Source, Argonne National Laboratory, USA. The specimens for the HEXRD were machined with the size of $7 \times 2 \times 0.6 \mathrm{~mm}^{3}$. X-ray perpendicular to the longitudinal section passed through the specimen to measure the internal residual stress. Fig. 1 shows the schematic diagram of the HEXRD measurement. The HEXRD beam with diameter of $0.1 \mathrm{~mm}$ was used to scan the specimens along the $z$ direction with step of $0.1 \mathrm{~mm}, d_{i}=(100 \cdot i-50) \mu \mathrm{m}(i=$ $1 \ldots 7)$ is the distance from the point center to the laser shocked surface, called depth in the following text (Fig. 1c); in each depth, 4 points with step of $0.1 \mathrm{~mm}$ along the $\mathrm{x}$ direction were scanned. A two-dimensional (2D) stationary detector with $200 \times 200 \mu \mathrm{m}^{2}$ pixel size was placed $1500 \mathrm{~mm}$ away from the specimen. Calibration was performed using $\mathrm{CeO}_{2}$ NIST powder standard.

For an isotropic glassy material without stress, the diffraction patterns are expected to be perfect circular haloes. By comparison, the elastic strain in BMGs under stress can be detected from the resulting elliptical distortion of the haloes [22]. The diffraction pattern is characterized by the polar coordinates $(r, \varphi)$, as shown in Fig. 1b. By dividing the $\varphi$ ranging from 0 to $2 \pi$ into 36 segments, the intensity distribution could be obtained as follows:

$I_{i}\left(Q, \varphi_{j}\right)=\int_{\varphi_{j}-\pi / 36}^{\varphi_{j}+\pi / 36} I_{i}(Q, \varphi) d \varphi$

where $j=1 \ldots 36, Q=Q(r)$ is defined by

$Q=4 \pi \sin (\theta) / \lambda$ 
where $\lambda$ is the wavelength of X-ray, $\theta$ is the Bragg angle, and $\theta=\arctan (r / D) / 2($ Fig. 1b). The intensity described by Eq. (1) was calculated using the software FIT 2D. The atomic level apparent strain caused by the applied stress can be calculated by the relative shift in the position of the first peak of $I_{i}\left(Q, \varphi_{j}\right)$, as given by

$\varepsilon_{i}\left(\varphi_{j}\right)=\frac{Q_{\max }^{0}\left(\varphi_{j}\right)-Q_{\max }^{i}\left(\varphi_{j}\right)}{Q_{\max }^{i}\left(\varphi_{j}\right)}$

where $Q_{\max }^{0}\left(\varphi_{j}\right)$ is the position of the first peak in the $I\left(Q, \varphi_{j}\right)$ measured on the sample without LSP process, and the $Q_{\max }^{i}\left(\varphi_{j}\right)$ is the position of the first peak in the $I_{i}\left(Q, \varphi_{j}\right)$

The angular variation of the strain can be fitted to the following expression:

$\varepsilon(\varphi)=\varepsilon_{x} \sin ^{2} \varphi+\gamma_{x z} \sin \varphi \cos \varphi+\varepsilon_{z} \cos ^{2} \varphi$

Thus, the strain in the $x$ direction $\varepsilon_{x}\left(\varphi=90^{\circ}\right)$ and in the $z$ direction $\varepsilon_{z}\left(\varphi=0^{\circ}\right)$ can be derived.

The SEM/FIB instrument (Tescan Lyra3) was used to measure the residual stress on the shocked surface of the LSPed specimen [16]. In the SEM/FIB instrument, the crack compliance (slitting by FIB, length: $30 \mu \mathrm{m}$, depth: $3.0 \mu \mathrm{m}$ ) method was used to detect the micro-scale residual stress. For an infinitely long crack in a thick plate, the displacement in the direction perpendicular to the slot can be given by the InglisMuskhelishvili solution [27]:

$u_{x t}=\frac{2.243}{E^{\prime}} \sigma \int_{0}^{a} \cos \theta\left(1+\frac{\sin ^{2} \theta}{2(1-v)}\right)(1.12+0.18 \operatorname{sech}(\tan \theta)) \mathrm{d} a$

where $E^{\prime}=E /\left(1-v^{2}\right)$, where $E$ is the Young's modulus and $v$ is the Poisson's ratio. $a$ is the slot depth, $x$ is the distance to the slot, $\theta=\arctan (x / a) . \sigma$ is the residual stress. By using the digital image correlation method to measure the released deformation, the residual stress normal to the slot can be obtained. The stress $\left(\sigma_{0}\right)$ on the surface of polished BMG was also measured. The detailed procedure could be found in Ref. [16]. 


\section{Results}

\subsection{Residual strain}

The 2D diffraction pattern (Fig. 2a) of the LSP-3 specimen exhibits a typical diffuse scattering pattern, confirming the amorphous structure without any crystallization after the LSP process. Fig. 2b shows the intensity spectra along the $x$ direction, i.e. the $I_{i}\left(Q, 90^{\circ}\right)(i=1,2,3)$ curves measured on the LSP-1 specimen. It has been proven that the first peak position would shift towards the lower $Q$ under tensile strain, and towards the higher $Q$ under compressive strain [28]. The inset of Fig. $2 \mathrm{~b}$ shows the shift of the first peak position corresponding to the different depths, indicating that the closer the measured points away from the shocked surface (smaller $\left.d_{i}\right)$ is, the larger the elastic compressive strain is. Fig. 2c shows plots of the first peak position vs. the circular coordinate $\varphi$. It could be found that the peak position varies with the change of $\varphi$, indicating that anisotropic elastic strain is generated in the LSPed specimen. Fig. 3a shows the plots of internal residual strain $\left(\varepsilon_{x}\right.$ and $\left.\varepsilon_{z}\right) v s$. depth measured on the LSP-1 specimen. Near the shocked surface, the $\varepsilon_{x}$ is much higher than the $\varepsilon_{z}$, indicating that the internal residual stress in the $x$ direction is much higher than that in the $z$ direction. Tensile strain began to appear when the depth is higher than $400 \mu \mathrm{m}$, which is consistent with the results reported by Fu et al. [15] and Greer et al. [18]. The $\varepsilon_{z}$ shows a notable phenomenon that the strain at lower depth is smaller than that at higher depth. The strain measured on the LSP-3 specimen is shown in Fig. 3b, indicating that the zone of $\varepsilon_{x}$ increased with the LSP cycle increase.

\subsection{Free volume}

Fig. 4 shows the DSC curves of the as-cast and the LSPed specimens. The structural relaxation enthalpy $(H)$ of the as-cast specimen $\left(H_{\text {as-cast }}\right)$ is $357.25 \mathrm{~J} / \mathrm{mol}$ and

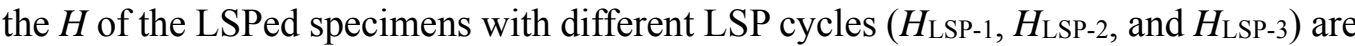
$775.25 \mathrm{~J} / \mathrm{mol}, 650.33 \mathrm{~J} / \mathrm{mol}$, and $580.44 \mathrm{~J} / \mathrm{mol}$, respectively. The correlation between the $H$ and the amount of free volume stored in BMGs was originally proposed by 
Sietsma et al. [29] which was experimentally demonstrated by Slipenyuk et al .[30] Launey et al. [31], and Evenson et al. [32].

According to Refs. [29-32], the change of the structural relaxation enthalpy $\Delta H$

$\Delta H=H_{\varepsilon \neq 0}-H_{\varepsilon=0}$

is related to the change of the relative free volume change $\Delta v_{f} / v_{m}$ by

$\Delta H=\beta \cdot \Delta v_{f} / v_{m}$

where $\varepsilon$ is the strain, $\beta$ is a constant, $\Delta v_{f}$ is the change of the free volume per atomic volume, and $v_{m}$ is the atomic volume. As a reference sample, the strain of the as-cast sample is assumed to be 0 , namely $H_{\text {as-cast }}=H_{\varepsilon=0}$. Slipenyuk et al. [30] obtained $\beta=552 \pm 15 \mathrm{KJ} / \mathrm{mol}$ for the $\mathrm{Zr}_{55} \mathrm{Cu}_{30} \mathrm{Al}_{10} \mathrm{Ni}_{5} \mathrm{BMG}$, and Evenson et al. [32] reported $\beta=623 \pm 20 \mathrm{KJ} / \mathrm{mol}$ for the $\mathrm{Zr}_{44} \mathrm{Ti}_{11} \mathrm{Ni}_{10} \mathrm{Cu}_{10} \mathrm{Be}_{25}$ BMG. Due to the similarity in composition between the present work and $\mathrm{Zr}_{44} \mathrm{Ti}_{11} \mathrm{Ni}_{10} \mathrm{Cu}_{10} \mathrm{Be}_{25} \mathrm{BMG}, \quad \beta=$ $600 \mathrm{KJ} / \mathrm{mol}$ is assumed to be a reasonable approximation in the present work $[6,26]$. Using $\beta=600 \mathrm{KJ} / \mathrm{mol}$ in Eq. (7), the excess free volume per atomic volume is estimated to be $0.070 \%, 0.049 \%$, and $0.037 \%$ for the LSP-1, LSP-2, and LSP-3 specimens, respectively. The results show that the free volume increased after the first LSP process, while the excess free volume did not continue to increase with additional LSP cycles, conversely, it decreased. The same phenomenon was reported by Huang et al. [33] and Flores et al. [34] while it is inconsistent with the works of Fornell et al. [3], Stolpe et al. [6], and Hajlaoui et al. [35].

\subsection{Hardness}

Fig. 5 shows the plots of hardness vs. depth on the longitudinal section of the LSPed specimens. As seen the plots of the LSP-1 specimen, a softening effect was present in the LSP-1 specimen: the hardness decreased obviously with the depth decrease, which is quite consistent with the results of the shot peened BMGs [3]. However, compared to the LSP-1 specimen, less softening effect is observed after LSP2 and even no softening for LSP-3 specimen.

The hardness distribution on the shocked surface of the LSP-1 specimen is shown in Fig. 6a. It was found that the hardness on the shocked surface center is greater than 
the hardness on the as-cast specimen, exhibiting hardening effect. Similar hardening effect was reported by Wei et al. [24]. For the LSP-2 and LSP-3 specimens, the hardness at the pit center is still higher than the as-cast sample but lower than that of the LSP-1 specimen, as shown in Fig. 6 b.

\section{Discussion}

\subsection{Residual stress}

\subsubsection{Internal residual stress}

Greer et al. [18] reported that the internal residual stress in the shot peened BMG is equiaxial plane stress on the $x-y$ plane (the coordinate system is shown in Fig. 1), namely $\sigma_{x}=\sigma_{y} \neq 0, \sigma_{z}=0$. The experiments and finite element modeling have proved that $\sigma_{x}$ and $\sigma_{y}$ are much higher than $\sigma_{z}$ [36,37], while there is no evidence indicating that $\sigma_{z}=0$. Thus, in the present work, the residual stress in the LSPed region is expected to be:

$\sigma_{x}=\sigma_{y} \neq 0, \sigma_{z} \neq 0$

However, because the specimens for the HEXRD were cut from the LSPed specimen (Fig. 1), the strain $\varepsilon_{y}$ and stress $\sigma_{y}$ are relaxed. The residual stress $\sigma_{y}$ is shown in Fig. 7, and the stress resultant of $\sigma_{y}$ is 0 . Thus, the residual stress in the specimen for the HEXRD is expected to be

$\sigma_{x} \neq 0, \sigma_{y}=0, \sigma_{z} \neq 0$

Using the generalized Hooke law, the strain is:

$\varepsilon_{x}=\frac{\sigma_{x}-v \sigma_{z}}{E}, \varepsilon_{z}=\frac{\sigma_{z}-v \sigma_{x}}{E}$,

thus the stress could be calculated using:

$\sigma_{x}=\frac{E}{1-v^{2}}\left(v \varepsilon_{z}+\varepsilon_{x}\right), \sigma_{z}=\frac{E}{1-v^{2}}\left(v \varepsilon_{x}+\varepsilon_{z}\right)$

where $v$ is the Poisson ratio, $E$ is the Young's modulus that measured to be $90 \pm 3 \mathrm{GPa}$.

Fig. 8 shows the residual stress as a function of depth on the longitudinal sections of the LSP-1 and LSP-3 specimens. The $\sigma_{x}$ is much higher than the $\sigma_{z}$ when the depth is low. For the LSP-1 specimen (Fig. 8a), the $\sigma_{x}$ decreased quickly with the depth increase, and transferred to be tensile stress when the depth is greater than 400 
$\mu \mathrm{m}$, which is quite consistent with the results of $\mathrm{Fu}$ et al. [25] and Cao et al. [17].

Different from $\sigma_{x}$, the decrease trend of the $\sigma_{z}$ is gentle, the $\sigma_{z}$ is still more than $100 \mathrm{MPa}$ when the depth is as high as $650 \mu \mathrm{m}$. When the LSP cycle increased from 1 to 3 , the depth of the compressive region in the $x$ direction increased from $400 \mu \mathrm{m}$ to $500 \mu \mathrm{m}$. The $\sigma_{x}$ near the shocked surface didn't increase with increasing the LSP cycles, the $\sigma_{x}$ on the LSP-3 specimen at the depth of $50 \mu \mathrm{m}$ is $-305 \mathrm{MPa}$ (Fig. 8b), slightly less than $-345 \mathrm{MPa}$ in the LSP-1 specimen (Fig. 8a).

During LSP loading, extreme high compressive stress was induced. The BMGs deformed within the LSP effect zone, introducing both elastic and plastic strain. The elastic strain caused ultra-high stress and the plastic strain lead to the generation of pit (Fig. 1a). The shocked surface is free in the $z$ direction, thus elastic deformation is easily recovered to zero and the absorbed elastic energy would be released after the laser shock, leaving only minor strain in the $z$ direction near the shocked surface (Fig. 3). However, on the $x-y$ plane, the elastic recovery is constrained by the surrounding material, thus elastic energy was successfully preserved. This gave rise to the obvious residual strain (Fig. 3) and the residual stress kept at relative high values (Figs. 8 and 9).

\subsubsection{Residual stress on the shocked surface}

To measure the residual stress distribution on the shocked surface, a SEM/FIB technology was used and the results were shown in Fig. 9. Fig. 9a exhibits the residual stress as a function of distance away from shock center for the LSP-1 specimen. The highest residual stress is about $-750 \mathrm{MPa}$. Fig. 9b shows the highest stress on the shocked surface with different LSP cycles. The residual stress slightly decreased with repeated LSP cycles, the highest stress on the shocked surface of the LSP-3 specimen is about $-600 \mathrm{MPa}$. The phenomenon that the stress decreased with increasing the LSP cycles is quite consistent with the stress distribution on the longitudinal section (Fig. 8).

Compared with the internal residual compressive stress (Fig. 8), the stress on the shocked surface is much higher. To further prove the phenomenon, a nanoindentation test was performed and the morphology of the indentations was characterized by AFM. 
Recently, Haag et al. [14] proved that residual compressive stress within BMGs is beneficial for the generation of pileups around the indentations. Figs. 10(a and b) show the AFM images of the indentations on the shocked surface and the longitudinal section $(d=50 \mu \mathrm{m})$, respectively. Fig. 10c shows the line profiles of the indentations. It could be found that pileups on the shocked surface are much more obvious than those on the longitudinal section, indicating a higher stress on the shocked surface.

\subsection{Free volume}

According to the DSC results, the maximum excess free volume value is observed in the LSP-1 specimen, and an increase in the LSP cycles (LSP-2 and LSP-3) caused a decrease in free volume compared to the LSP-1 (Fig. 4). However, it should be noted that the excess free volume of the LSP-3 specimen is still higher than that of the as-cast specimen, indicating that the deformation did in fact increase the free volume, which is consistent with the results of Huang et al. [33] and Flores et al. [34].

There are two processes determining the free volume variation of the deformed BMG: the free volume generation induced by plastic deformation and the free volume annihilation (structural relaxation) induced by diffusion atomic jumps [38]. Due to the relative high peak pressure of the LSP, the BMG deformed severely within localized region during the LSP-1 process. The applied stress, especially the shear stress, could force a few atoms into the neighboring holes whose volume is smaller than those atoms themselves, thus the excess free volume is generated [7]. During the LSP-2 and LSP-3 processes, the increased free volume promoted more atomic jumps [33] resulting in that the annihilation defeated the generation of free volume. Finally the generation and annihilation of the free volume achieved dynamic balance, and the excess free volume was kept steady $[33,34]$. Thus, it is suggested that the free volume induced in previous deformation would annihilate during subsequent deformation, showing a self-repairing dynamic recovery process [39]. However, the above speculation needs further evidence and better theory to stand. Flores et al. [34] obtained a similar result during investigating the cold-rolled $\mathrm{Zr}_{58.5} \mathrm{Cu}_{15.6} \mathrm{Ni}_{12.8} \mathrm{Al}_{10.3} \mathrm{Nb}_{2.8}$ BMG. However, Stolpe et al. [6] found that the free volume monotonously increased with the strain increase by cold- 
rolling, contrary to the work of the authors and Flores et al. [34]. The inconsistency means that further research is needed.

\subsection{Influence of free volume and residual stress on hardness}

It has been proved that the promotion of free volume could decrease hardness, causing a softening effect [9-11]; while the residual compress stress could increase hardness by inhibiting the propagation of shear bands, causing a hardening effect $[13,14]$. The softening effect was found on the longitudinal section of the LSPed specimens (Fig. 5), and the hardening effect was found on the shocked surface (Fig. 6a). It is supposed that both the softening mechanism by free volume and the hardening mechanism by residual compressive stress influenced the hardness of the LSPed BMGs. The main difference between the longitudinal section and the shocked surface is that the internal residual stress is much lower than the stress on the shocked surface (Figs. 8-10). The minimum depth (Fig. 8) for measuring hardness on the longitudinal section is about $50 \mu \mathrm{m}$, where the internal residual stress $\left(\sigma_{x}=-345 \mathrm{MPa}\right)$ is much lower than the residuals stress at the center of the pit $\left(\sigma_{x}=-750 \mathrm{MPa}\right)$ on the shocked surface (Fig. 9). Moreover, internal residual stress also showed the anisotropy (Fig. 8), i.e. the $\sigma_{z}$ is much lower than $\sigma_{x}$. The low internal residual stress has little effect on the hardness increase, and the hardness decrease induced by excess free volume plays the principal role on the longitudinal section, causing the softening effect. However, the stress on the shocked surface is extremely high and isotropic, which plays the principal role on the influence of hardness, resulting in the final hardening effect.

By analyzing the LSP-2 and LSP-3 specimens, it could be found that the softening effect on the longitudinal section was weakened with the LSP cycle increase (Fig. 5). Figs. 4 and 8 show that the free volume decreased after LSP-2 and LSP-3, while the residual stress had nearly no change in comparison with the LSP-1 specimen, indicating that the softening was dampened due to the free volume decrease. The maximum hardness on the shocked surface also decreased with increasing LSP cycles (Fig. 6b), resulting in the undermined inhibition of shear bands propagation in the LSP-2 or LSP3 specimens. Thus, the hardening effect in LSP-2 and LSP-3 specimens was reduced. 
By comparing the hardness results of the LSPed specimens, it could be found that the increased LSP cycles not only increased the residual compression zone, but also had a recover influence on the structure of BMGs. It is assumed that the free volume and residual elastic deformation in the effect zone is oversaturated and unstable after the first LSP. Due to the extremely short loading time during the LSP (less than $100 \mathrm{~ns}$ ) and the constraint of the surrounding material, the deformation was inhomogeneous and amounts of oversaturated regions were preserved under the shocked surface. During the subsequent LSP, the energy in the oversaturated regions was relaxed, thus the annihilation rate of the oversaturated regions was higher than the generation rate, the net energy was reduced and the structure tended towards stability.

\section{Conclusion}

Based on the study of the residual stress, free volume, and hardness of the LSPed BMGs, the following conclusions can be obtained.

(1) Under the shocked surface of the LSPed specimen, when the depth is small, the anisotropy of the residual stress is quite obvious, the residual compressive stress parallel with to shocked surface is nearly twice as high as that perpendicular to the shocked surface. Compared to the residual stress on the shocked surface, the internal residual stress is much lower. The increased LSP cycles cause step-up of the depth of the compressive stress zone.

(2) The free volume was vastly increased in the BMG after the first LSP, and then decreased after the second and third LSP. It is supposed that the free volume was oversaturated after the first LSP and the free volume annihilation became more significant during subsequent LSPs.

(3) Hardness of the specimens was determined by the softening mechanism caused by free volume and the hardening effect caused by the residual compressive stress under a competitive basis. The free volume played a principal role on the longitudinal section,

thus the softening effect was observed. However, the residual compressive stress played a principal role on the shocked surface, thus the hardening effect was observed. Both kinds of effects were weakened with the increased LSP cycles. 


\section{Acknowledgements}

The authors gratefully acknowledge the financial support of the National Natural Science Foundation of China (No. 51471035), Beijing Higher Education Young Elite Teacher Project, and the Program of "One Hundred Talented People" of the Chinese Academy of Sciences. Use of the Advanced Photon Source was supported by the U.S. Department of Energy, Office of Science, Office of Basic Energy Science, under Contract No. DE-AC02-06CH11357.

\section{References}

[1] A. Inoue, Stabilization of Metallic Supercooled Liquid, Acta Mater. 48 (2000) 279-306. doi:10.1016/S1359-6454(99)00300-6.

[2] Y. Zhang, W.H. Wang, A.L. Greer, Making metallic glasses plastic by control of residual stress, Nat. Mater. 5 (2006) 857-60. doi:10.1038/nmat1758.

[3] J. Fornell, A. Concustell, A.L. Greer, S. Suriñach, M.D. Baró, J. Sort, Effects of shot peening on the nanoindentation response of $\mathrm{Cu} 47.5 \mathrm{Zr} 47.5 \mathrm{Al} 5$ metallic glass, J. Alloys Compd. 586 (2014) S36-S40. doi:10.1016/j.jallcom.2012.12.051.

[4] W.H. Jiang, M. Atzmon, Room-temperature flow in a metallic glass - Strain-rate dependence of shear-band behavior, J. Alloys Compd. 509 (2011) 7395-7399. doi:10.1016/j.jallcom.2011.04.041.

[5] K.K. Song, S. Pauly, Y. Zhang, S. Scudino, P. Gargarella, K.B. Surreddi, et al., Significant tensile ductility induced by cold rolling in $\mathrm{Cu} 47.5 \mathrm{Zr}_{47.5} \mathrm{~A}_{15}$ bulk metallic glass, Intermetallics. 19 (2011) 1394-1398. doi:10.1016/j.intermet.2011.05.001.

[6] M. Stolpe, J.J. Kruzic, R. Busch, Evolution of shear bands, free volume and hardness during cold rolling of a Zr-based bulk metallic glass, Acta Mater. 64 (2014) 231-240. doi:10.1016/j.actamat.2013.10.035.

[7] F. Spaepen, A microscopic mechanism for steady state inhomogeneous flow in metallic glasses, Acta Metall. 25 (1977) 407-415. doi:10.1016/0001-6160(77)90232-2. 
[8] A.S. Argon, Plastic deformation in metallic glasses, Acta Metall. 27 (1979) 47-58. doi:10.1016/0001-6160(79)90055-5.

[9] E.M. Axinte, Y. Wang, L.L. Tabacaru, N. Radwan, Shear Banding in Metallic Glasses: Major Weakness or Potential Advantage?, Recent Patents Mater. Sci. 9 (2016) $2-19$.

[10] J. Tan, Y. Zhang, B.A. Sun, M. Stoica, C.J. Li, K.K. Song, U. Kühn, F.S. Pan, J. Eckert, Correlation between internal states and plasticity in bulk metallic glass, Appl. Phys. Lett. 98 (2011)151906. doi:10.1063/1.3580774.

[11]L.Y. Chen, A.D. Setyawan, H. Kato, A. Inoue, G.Q. Zhang, J. Saida, et al., Freevolume-induced enhancement of plasticity in a monolithic bulk metallic glass at room temperature, Scr. Mater. 59 (2008) 75-78. doi:10.1016/j.scriptamat.2008.02.025.

[12]H. Bei, S. Xie, E. George, Softening Caused by Profuse Shear Banding in a Bulk $\begin{array}{lllllll}\text { Metallic Glass, } & \text { Phys. } & \text { Rev. } & \text { Lett. } 96 & \text { (2006) }\end{array}$ doi:10.1103/PhysRevLett.96.105503.

[13]L. Wang, H. Bei, Y.F. Gao, Z.P. Lu, T.G. Nieh, Effect of residual stresses on the hardness of bulk metallic glasses, Acta Mater. 59 (2011) 2858-2864. doi:10.1016/j.actamat.2011.01.025.

[14]F. Haag, D. Beitelschmidt, J. Eckert, K. Durst, Influences of residual stresses on the serrated flow in bulk metallic glass under elastostatic four-point bending - A nanoindentation and atomic force microscopy study, Acta Mater. 70 (2014) 188-197. doi:10.1016/j.actamat.2014.01.053.

[15]L.Z. Zhao, R.J. Xue, Z.G. Zhu, Z. Lu, E. Axinte, W.H. Wang, H.Y. Bai, Evaluation of flow units and free volumes in metallic glasses, J. Appl. Phys. 116 (2014) 103516. doi:10.1063/1.4895586.

[16]R. Zhu, H. Xie, Y. Xue, L. Wang, Y. Li, Fabrication of speckle patterns by focused ion beam deposition and its application to micro-scale residual stress measurement, Meas. Sci. Technol. 26 (2015) 095601. doi:10.1088/0957-0233/26/9/095601. 
[17]Y. Cao, X. Xie, J. Antonaglia, B. Winiarski, G. Wang, Y.C. Shin, P.J. Withers, K.A. Dahmen, P.K. Liaw, Laser Shock Peening on Zr-based Bulk Metallic Glass and Its Effect on Plasticity: Experiment and Modeling, Sci. Rep. 5 (2015) 10789. doi:10.1038/srep10789.

[18]F.O. Méar, G. Vaughan, A.R. Yavari, A.L. Greer, Residual-stress distribution in shot-peened metallic-glass plate, Philos. Mag. Lett. 88 (2008) 757-766. doi:10.1080/09500830802235776.

[19]A.J.G. Lunt, A.M. Korsunsky, A review of micro-scale focused ion beam milling and digital image correlation analysis for residual stress evaluation and error estimation, Surf. Coatings Technol. 283 (2015) 373-388. doi:10.1016/j.surfcoat.2015.10.049.

[20]Y. Li, K. Georgarakis, S. Pang, C. Ma, G. Vaughan, A.R. Yavari, et al., Real time synchrotron radiation studies on metallic glass $\left(\mathrm{Zr}_{0} .55 \mathrm{~A}_{10.1} \mathrm{Ni}_{0.05} \mathrm{Cu}{ }_{0.3}\right){ }_{99} \mathrm{Y}_{1}$ after cold rolling, Intermetallics. 17 (2009) 231-234. doi:10.1016/j.intermet.2008.08.015.

[21]H.S. Shahabi, S. Scudino, I. Kaban, M. Stoica, U. Rütt, U. Kühn, et al., Structural aspects of elasto-plastic deformation of a Zr-based bulk metallic glass under uniaxial compression, Acta Mater. 95 (2015) 30-36. doi:10.1016/j.actamat.2015.05.011.

[22]H.F. Poulsen, J.A. Wert, J. Neuefeind, V. Honkimaki, M. Daymond, Measuring strain distributions in amorphous materials, Nat. Mater. 4 (2005) 33-36. doi:10.1038/nmat1266.

[23]L.Y. Chen, B.Z. Li, X.D. Wang, F. Jiang, Y. Ren, P.K. Liaw, et al., Atomic-scale mechanisms of tension-compression asymmetry in a metallic glass, Acta Mater. 61 (2013) 1843-1850. doi:10.1016/j.actamat.2012.11.054.

[24]G.Y. Xu, Y.P. Wei, B.C. Wei, Deformation feature and properties of Zr based bulk metallic glass treated by laser shock peening, Mater. Res. Innov. 18S4 (2014) 798-802. doi:10.1179/1432891714Z.000000000783.

[25]J. Fu, Y. Zhu, C. Zheng, R. Liu, Z. Ji, Finite element simulation of laser shock peening on Bulk Metallic Glass, Appl. Surf. Sci. 313 (2014) 692-697. doi:10.1016/j.apsusc.2014.06.056. 
[26]L. Wang, L. Wang, Y. Xue, H. Zhang, H. Fu, Nanoindentation response of laser shock peened Ti-based bulk metallic glass, AIP Adv. 5 (2015) 057156. doi:10.1063/1.4921898.

[27]B. Winiarski, R.M. Langford, J. Tian, Y. Yokoyama, P.K. Liaw, P.J. Withers, Mapping residual stress distributions at the micron scale in amorphous materials, Metall. Mater. Trans. A Phys. Metall. Mater. Sci. 41 (2010) 1743-1751. doi:10.1007/s11661009-0127-4.

[28]M. Stoica, J. Das, J. Bednarcik, H. Franz, N. Mattern, W.H. Wang, et al., Strain distribution in $\mathrm{Zr}_{64.13} \mathrm{Cu}_{15.75} \mathrm{Ni}_{10.12} \mathrm{Al}_{10}$ bulk metallic glass investigated by in situ tensile tests under synchrotron radiation, J. Appl. Phys. $104 \quad$ (2008) 013522. doi:10.1063/1.2952034.

[29]A. van den Beukel, J. Sietsma, The glass transition as a free volume related kinetic phenomenon, Acta Metall. Mater. 38 (1990) 383-389. doi:10.1016/09567151(90)90142-4.

[30] A. Slipenyuk, J. Eckert, Correlation between enthalpy change and free volume reduction during structural relaxation of $\mathrm{Zr}_{55} \mathrm{Cu}_{30} \mathrm{Al}_{10} \mathrm{Ni}$ is metallic glass, Scr. Mater. 50 (2004) 39-44. doi:10.1016/j.scriptamat.2003.09.038.

[31]M.E. Launey, J.J. Kruzic, C. Li, R. Busch, Quantification of free volume differences in a $\mathrm{Zr}_{44} \mathrm{Ti}_{11} \mathrm{Ni}_{10} \mathrm{Cu}_{10} \mathrm{Be}_{25}$ bulk amorphous alloy, Appl. Phys. Lett. 91 (2007) 051913. doi:10.1063/1.2766659.

[32]Z. Evenson, R. Busch, Enthalpy recovery and free volume relaxation in a $\mathrm{Zr}_{44} \mathrm{Ti}_{11} \mathrm{Ni}_{10} \mathrm{Cu}_{10} \mathrm{Be}_{25}$ bulk metallic glass, J. Alloys Compd. 509 (2011) S38-S41. doi:10.1016/j.jallcom.2010.12.044.

[33]Y. Huang, Y. Sun, J. Shen, Tuning the mechanical performance of a Ti-based bulk metallic glass by pre-deformation, Intermetallics. 18 (2010) 2044-2050. doi:10.1016/j.intermet.2010.06.006. 
[34] K.M. Flores, E. Sherer, A. Bharathula, H. Chen, Y.C. Jean, Sub-nanometer open volume regions in a bulk metallic glass investigated by positron annihilation, Acta Mater. 55 (2007) 3403-3411. doi:10.1016/j.actamat.2007.01.040.

[35]K. Hajlaoui, T. Benameur, G. Vaughan, A.R. Yavari, Thermal expansion and indentation-induced free volume in Zr-based metallic glasses measured by real-time diffraction using synchrotron radiation, Scr. Mater. 51 (2004) 843-848. doi:10.1016/j.scriptamat.2004.07.008.

[36]A.F.M. Arif, Numerical prediction of plastic deformation and residual stresses induced by laser shock processing, J. Mater. Process. Technol. 136 (2003) 120-138. doi:10.1016/S0924-0136(02)01122-6.

[37]C. Correa, A. Gil-Santos, J.A. Porro, M. Díaz, J.L. Ocaña, Eigenstrain simulation of residual stresses induced by laser shock processing in a Ti6A14V hip replacement, Mater. Des. 79 (2015) 106-114. doi:10.1016/j.matdes.2015.04.048.

[38]Y. Zhang, Inhomogeneous deformation in metallic glasses, Mater. Sci. Technol. 24 (2002) 379-391. doi:10.1179/174328408X276044.

[39] Y.C. Lo, H.S. Chou, Y.T. Cheng, J.C. Huang, J.R. Morris, P.K. Liaw, Structural relaxation and self-repair behavior in nano-scaled $\mathrm{Zr}-\mathrm{Cu}$ metallic glass under cyclic loading: Molecular dynamics simulations, Intermetallics. 18 (2010) 954-960. doi:10.1016/j.intermet.2010.01.012.

\section{Figure captions:}

Fig. 1. (a) The scheme of the specimen for the HEXRD, (b) the experimental set-up of the HEXRD, (c) and the scanned points.

Fig. 2. (a) The 2D diffraction pattern of the LSP-3 specimen; (b) the typical scattering intensity spectra along the $x$ direction, i.e. $I_{i}\left(\mathrm{Q}, 90^{\circ}\right)(i=1,2,3)$, with an inset showing the shift of the first peak positions; (c) the angular dependence of the first peak position determined at different depths. 
Fig. 3. The relationship between the residual strain and the depth in the $x$ and $z$ directions ( $\varepsilon_{x}$ and $\varepsilon_{z}$ ) measured on the LSP-1 (a) and LSP-3 (b) specimens, respectively.

Fig. 4. DSC curves of the as-cast and LSPed specimens (Insets: the structural relaxation enthalpy $H$ as a function of the LSP cycle number and the schematic of the specimens for DSC).

Fig. 5. The plots of hardness vs. depth on the longitudinal section of the LSPed specimens.

Fig. 6. (a) The plots of hardness vs. the distance away from the center of the shocked surface for the LSP-1 specimen, the red shade area is the hardness on the as-cast BMG surface $(567 \pm 7 \mathrm{HV})$; (b) the maximum hardness on the shocked surface as a function of the LSP cycle number.

Fig. 7. The distribution of the residual stress along the $y$ direction $\left(\sigma_{y}\right)$ for the HEXRD specimen.

Fig. 8. The relationship between the residual stress and the depth in the $x$ and $z$ directions ( $\sigma_{x}$ and $\sigma_{z}$ ) measured on the LSP-1 (a) and LSP-3 (b) specimens, respectively.

Fig. 9. (a) The residual stress on the shocked surface of the LSP-1 specimen; (b) the highest residual stress on the shocked surface as a function of the LSP cycle number. Fig. 10. The morphologies of the indentations at the center of the shocked surface (a) and the location at depth of $50 \mu \mathrm{m}$ on the longitudinal section (b), respectively; (c) the line profiles of the indentations in (a) and (b). 
Figure 1

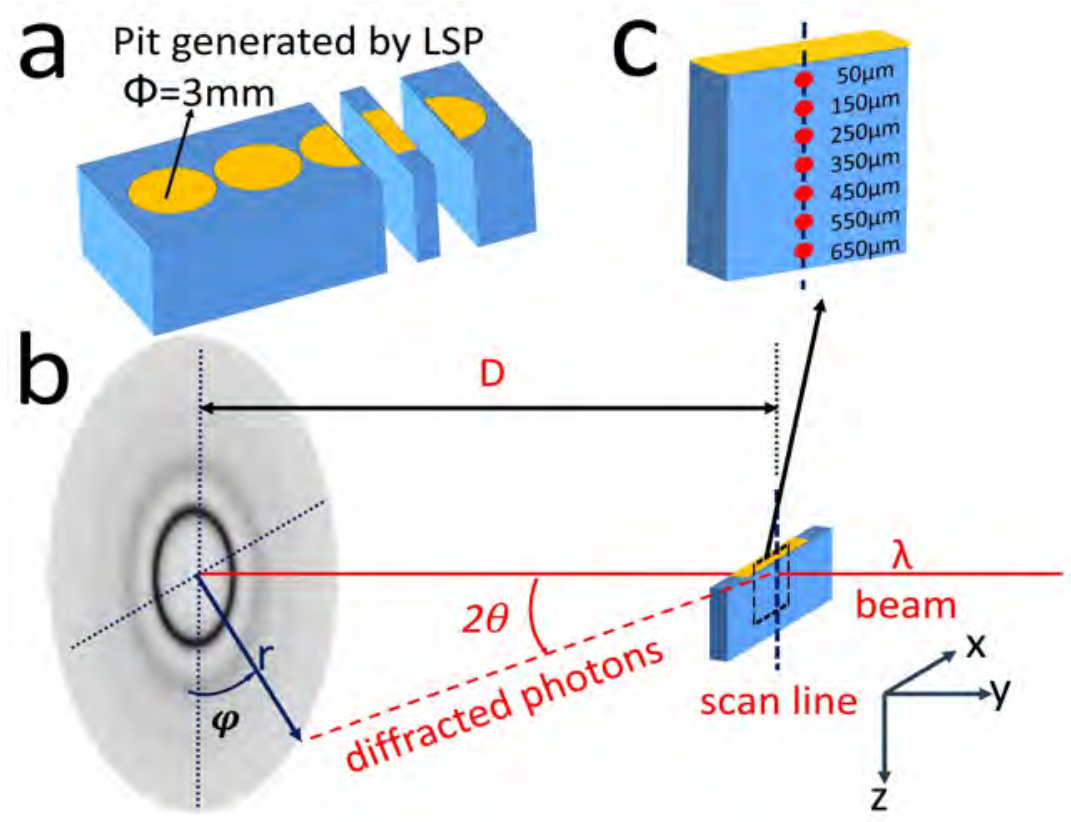


Figure 2
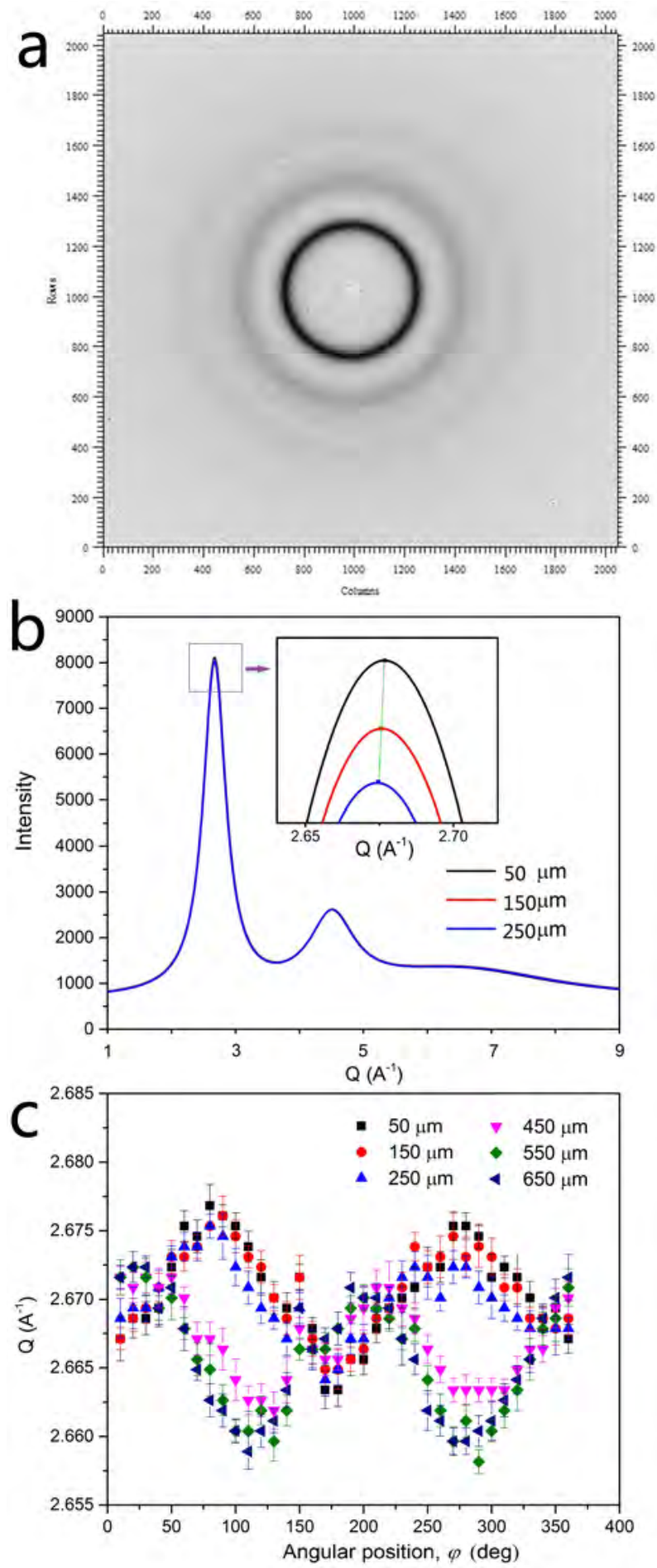
Figure 3
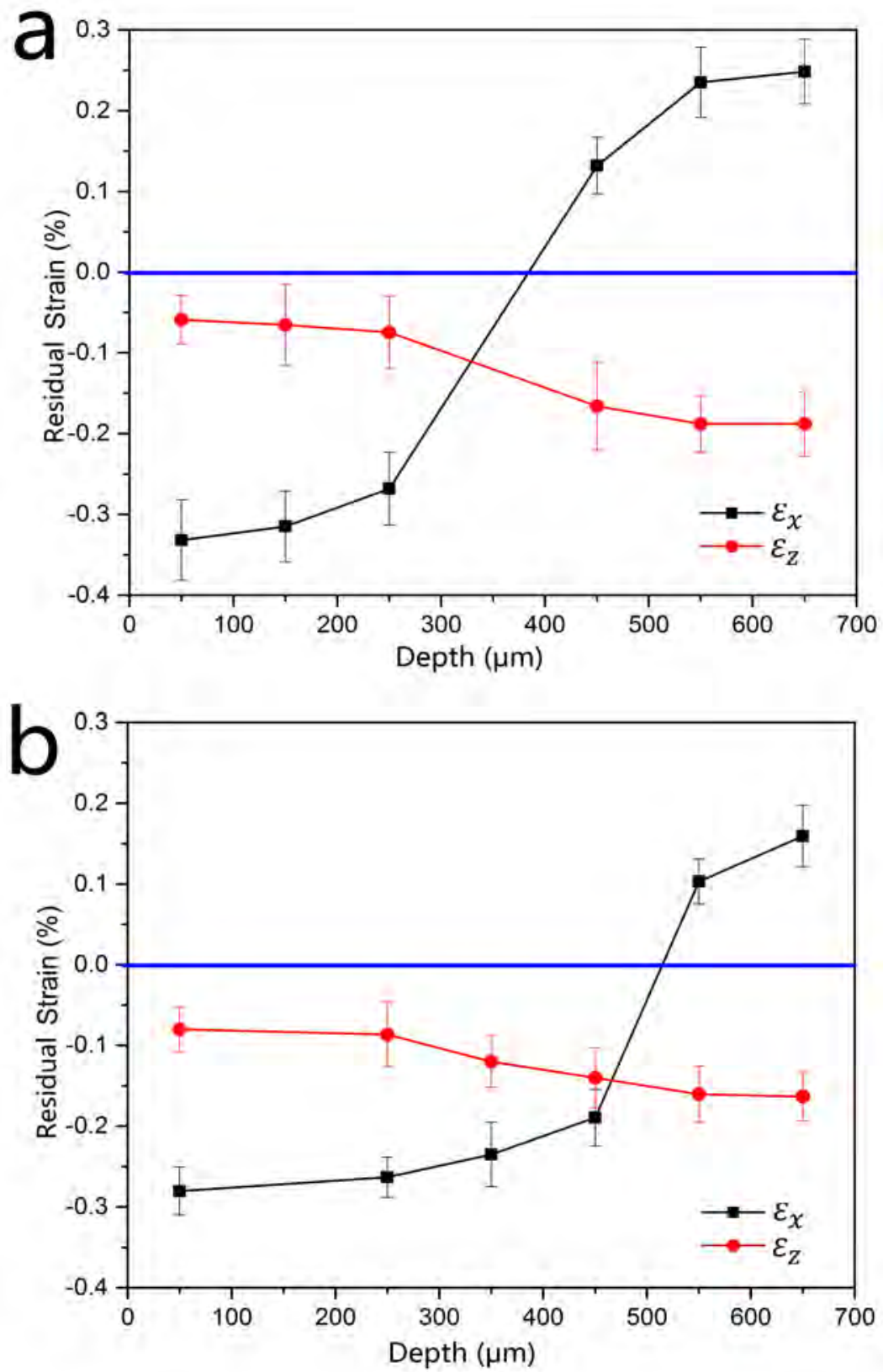
Figure 4

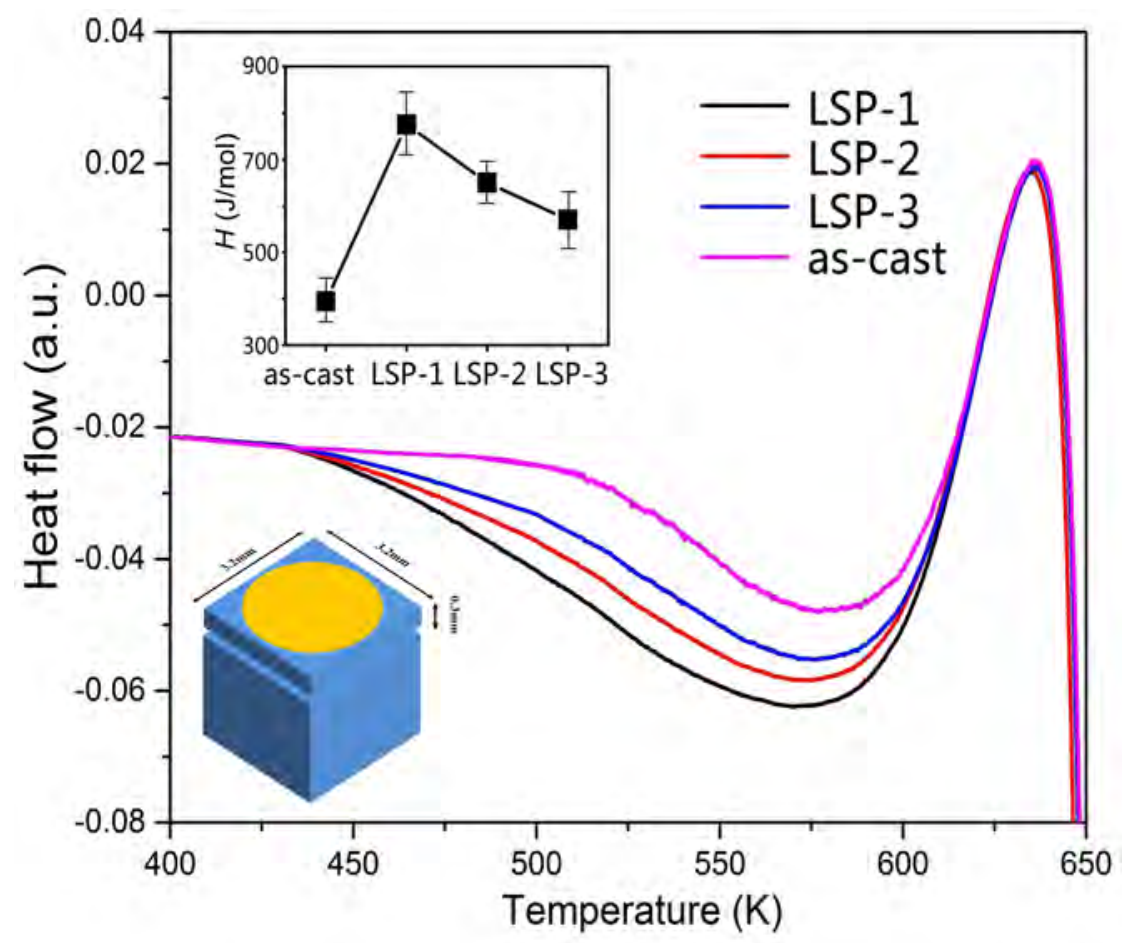


Figure 5

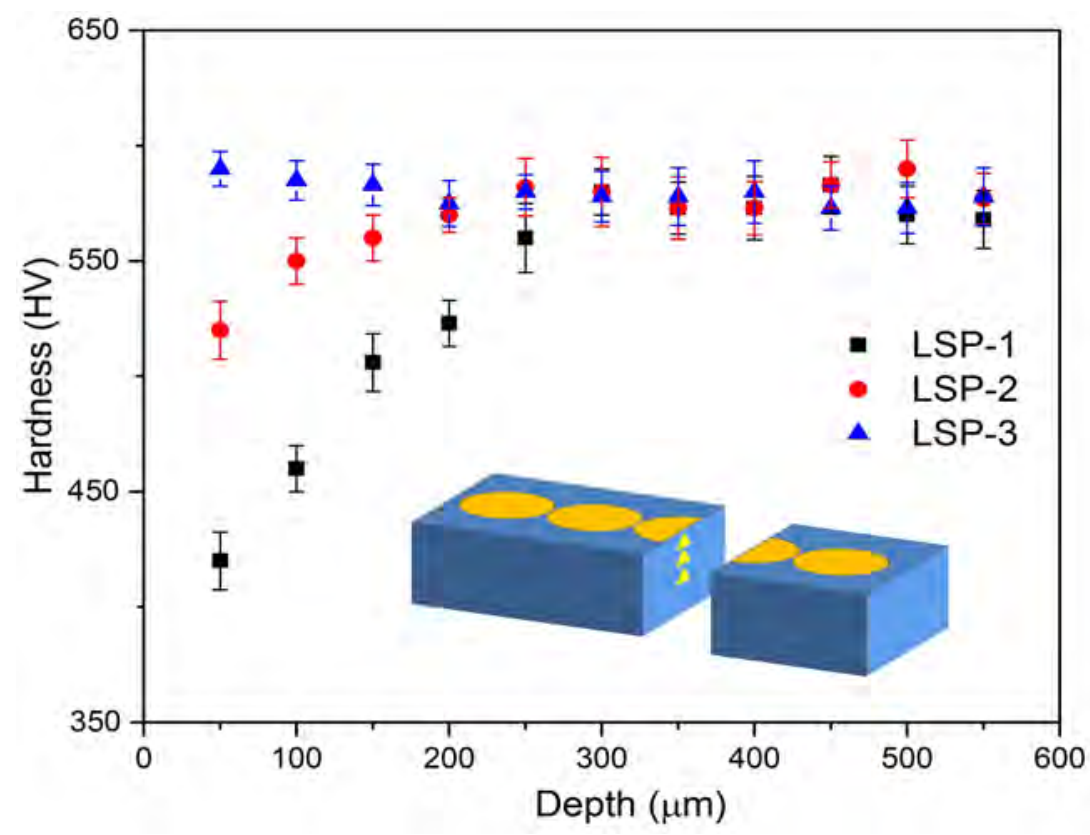


Figure 6
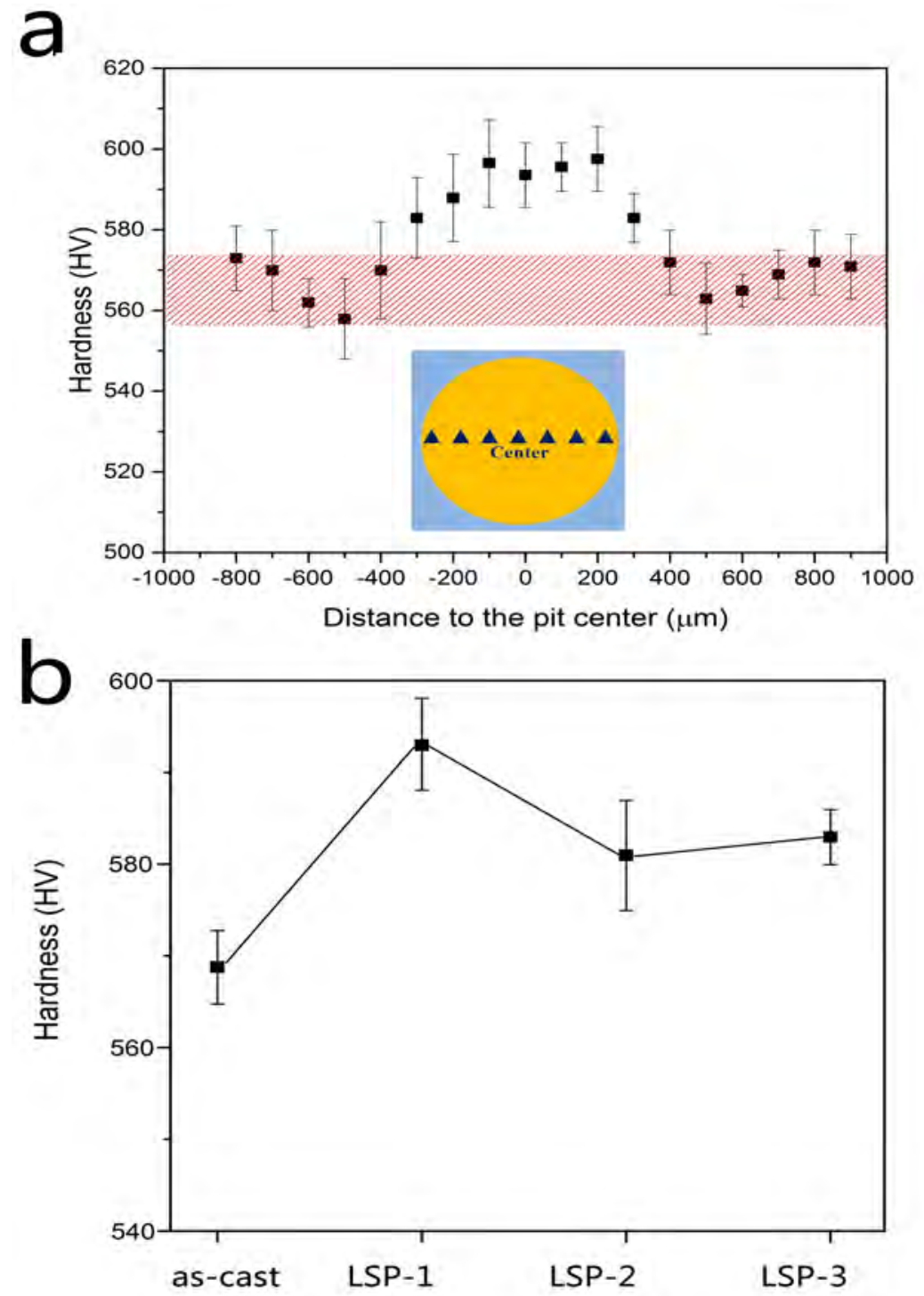
Figure 7

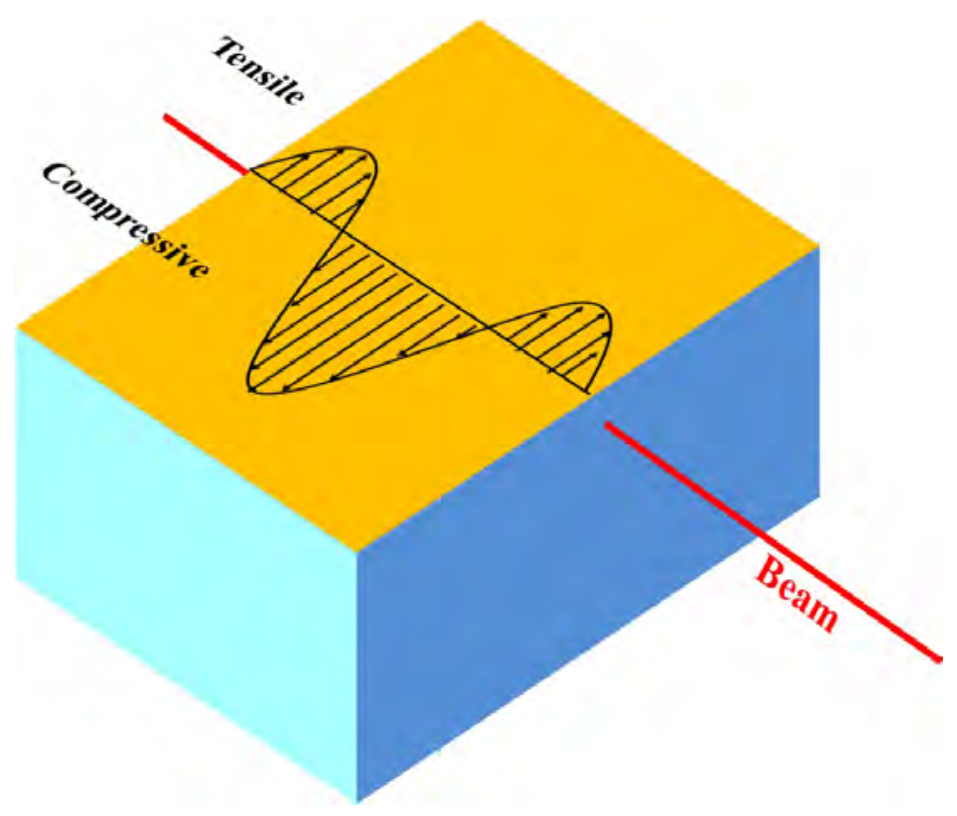


Figure 8
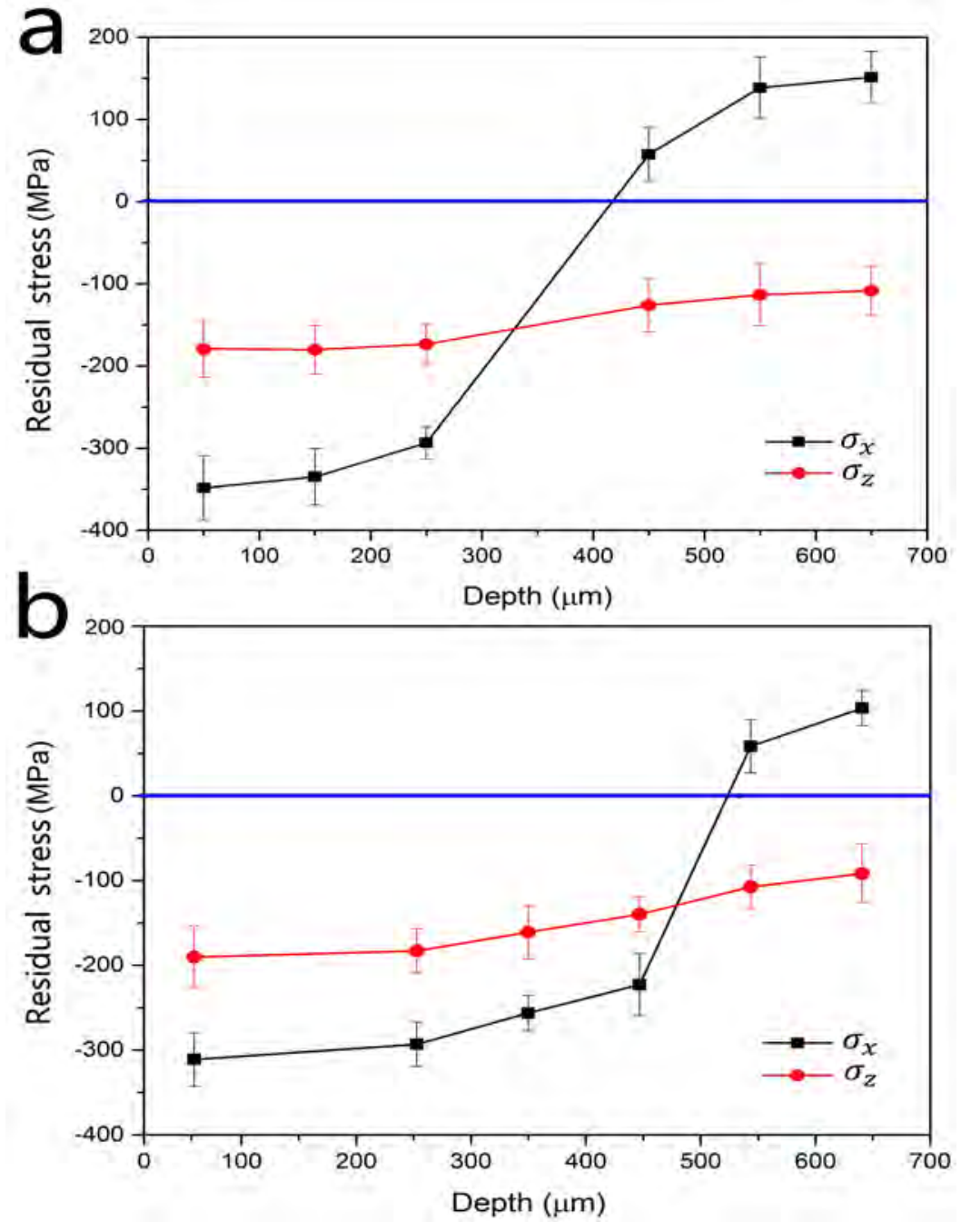
Figure 9
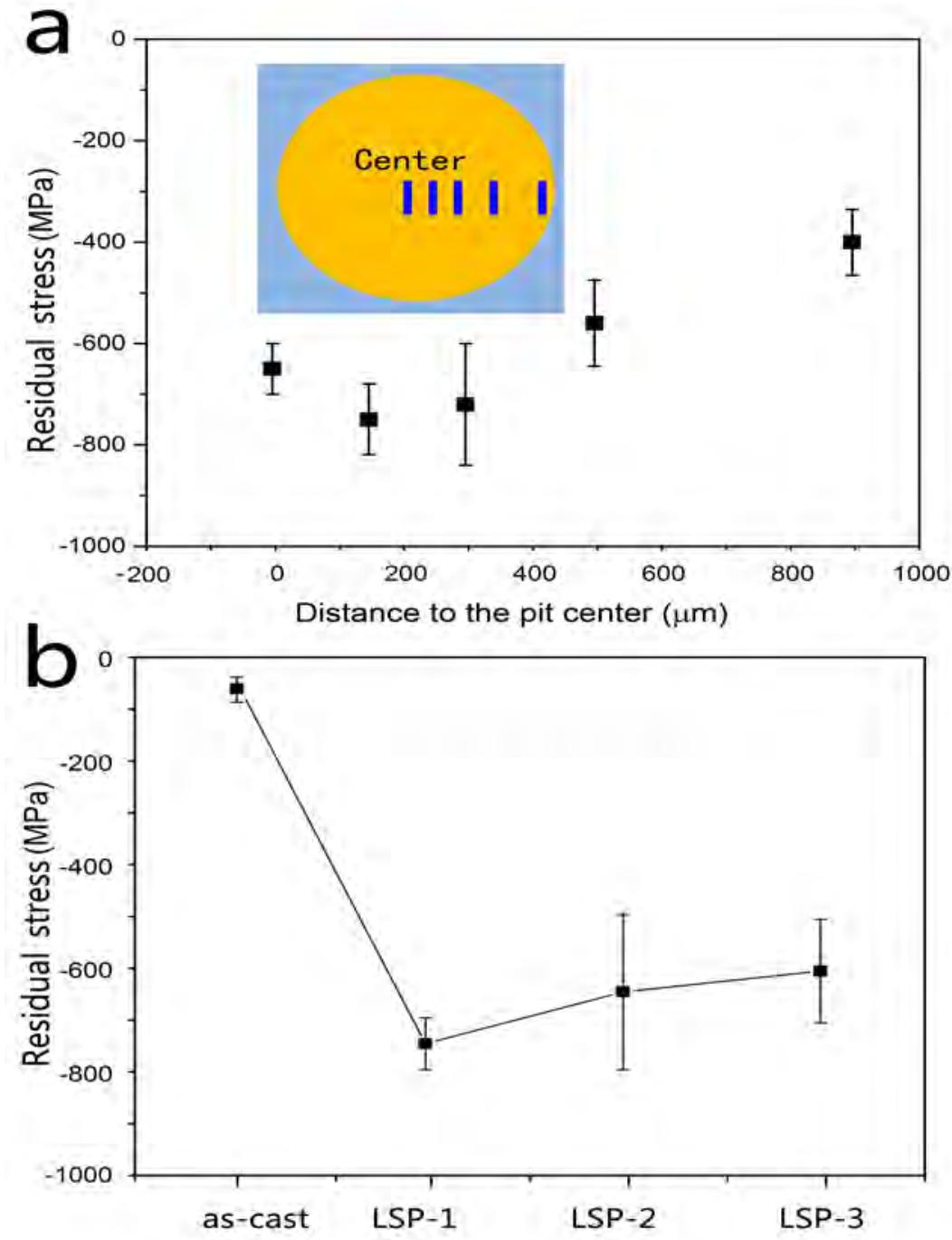
Figure 10
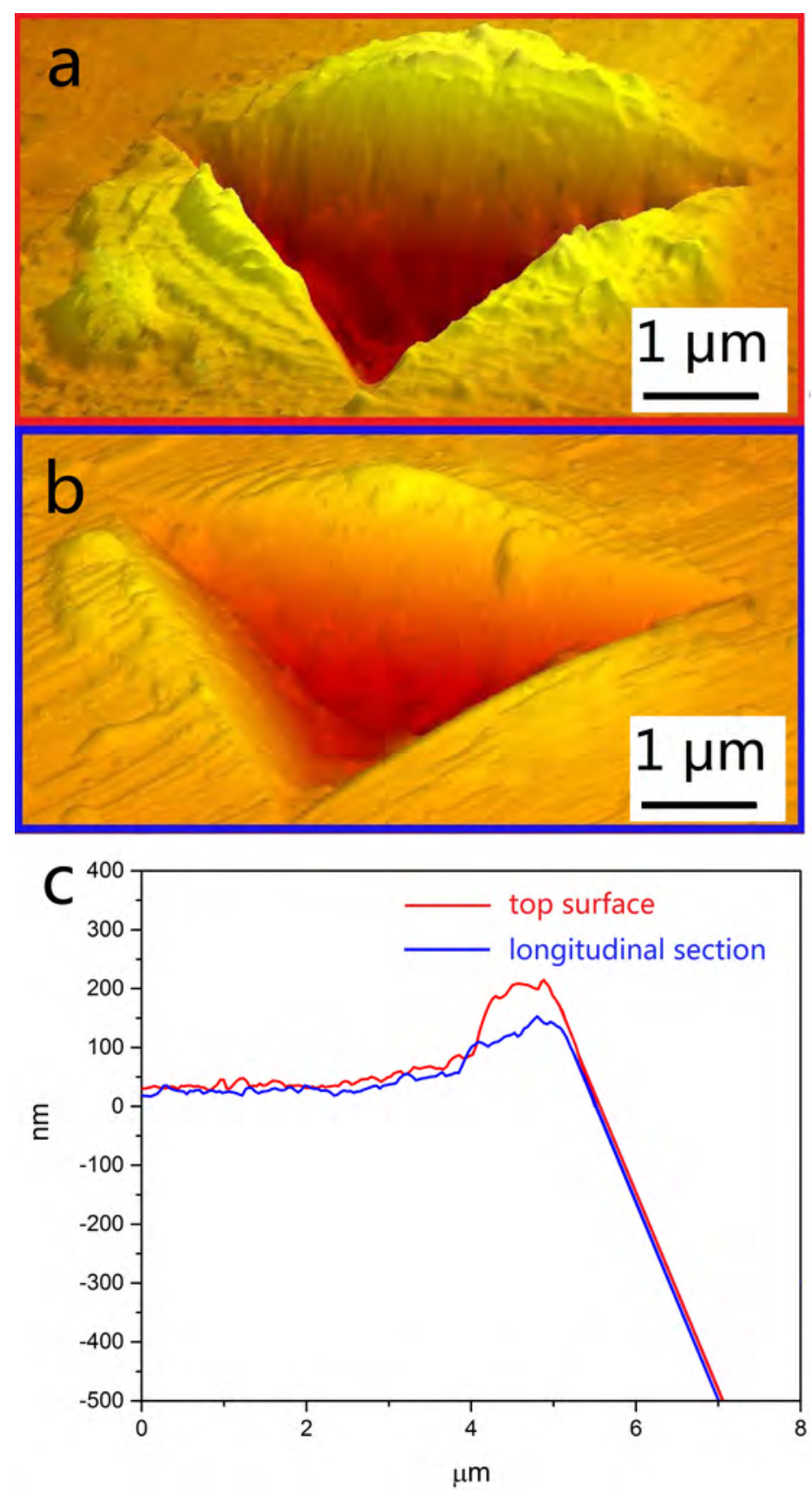